\title{
Relación entre marketing interno y el compromiso organizacional de los trabajadores de una empresa pública de Lima
}

\author{
Relationship between internal marketing and organizational commitment of the \\ employees of a public enterprise of Lima \\ Manuel Torres V. ${ }^{1}$, Manuel Torres L. \\ Universidad Nacional Mayor de San Marcos. Lima, Perú \\ (RECIBIDO 07/01/2014, ACEPTADO 24/03/2014)
}

\begin{abstract}
RESUMEN
El propósito fundamental del presente trabajo de investigación fue obtener una descripción de cómo perciben los trabajadores de una empresa pública de Lima el marketing interno que se desarrolla en ella y como este se relaciona con su compromiso organizacional. Se utilizó un diseño descriptivo correlacional, con una muestra de 279 trabajadores a quienes se les aplicó dos instrumentos de evaluación: el cuestionario de marketing interno, de María Bohnenberger, y el inventario de compromiso organizacional, de Allen y Meyer. Estos instrumentos fueron sometidos a los análisis respectivos que determinaron que las pruebas son válidas y confiables.

Los resultados indican que existen correlaciones significativas y positivas entre el marketing interno y el compromiso organizacional en esta muestra de trabajadores $(r=0.79)$. Asimismo se encontró que las trabajadoras alcanzan puntajes más altos, tanto en el marketing interno como en el compromiso organizacional, que los trabajadores varones.
\end{abstract}

Palabras clave: marketing interno, compromiso organizacional, clima organizacional, satisfacción laboral

\begin{abstract}
The main purpose of the current investigation was to obtain a description of how the employees of a public enterprise of Lima perceive the internal marketing that develops on it and how this relates to their organizational commitment. A correlational descriptive design was used with a sample of 279 employees to whom two instruments of evaluation were applied: the Internal Marketing Questionnaire of Maria Bohnenberger and the Inventory of Organizational Commitment of Allen and Meyer. These instruments were submitted to the respective analyses that determined that the tests are valid and reliable.
\end{abstract}


RELACIÓN ENTRE MARKETING INTERNO Y EL COMPROMISO ORGANIZACIONAL DE LOS TRABAJADORES DE UNA EMPRESA PÚBLICA DE LIMA

The results show that significant and positive correlations exist between internal marketing and organizational commitment in this sample of workers $(\mathrm{r}=0.79)$. Moreover, it was found that the female workers obtain higher scores than the male workers in both internal marketing and organizational commitment.

Keywords: Internal Marketing, Organizational Commitment, Organizational climate, labour satisfaction.

\section{INTRODUCCIÓN}

Atrás han quedado los tiempos en los que el empleador exigía de sus empleados esfuerzo de producción; la creatividad y la autonomía eran aspectos inapropiados para estar en la organización. Para lo cual, la jerarquización en mandos y supervisión de la tarea se asentaba donde la comunicación interna era inexistente, bastaba una orden para obtener el cumplimiento sin queja o solicitud alguna. El trabajo era de reconocimiento individual, la estructura salarial era rígida, se descontaba el ausentismo, la rotación era escasa, la formación del personal era vista como innecesaria, a menos que fuese requerida por los cambios tecnológicos.

Pero toda esta forma de gestionar al personal ha debido ser reevaluada a causa de las diversas crisis que han atravesado las organizaciones. Se hizo necesario ver a cada uno de los trabajadores como colaboradores de quienes dependían los resultados finales y entender que el vínculo de este con la empresa era esencial a la hora de analizar su nivel de rendimiento y productividad.

Es así como las empresas comenzaron a diagnosticarse interiormente, viendo al personal de la empresa como su cliente interno, hacia el cual, por su importancia, era necesario encaminar medidas que instauren una relación estrecha para lograr en él una visión de satisfacción y espejo proyectivo al exterior sobre la imagen no solo del producto, sino de la compañía, beneficios, políticas, cultura, gestión, etc.

Llegar a reconocer en el capital humano la ventaja competitiva no ha sido una tarea fácil, lo que conlleva mirar a cada miembro que integra la empresa como la representación de un cliente en quien debemos establecer también objetivos alineados a la estrategia empresarial. Esto es lo que se llama marketing interno.

El marketing interno, es decir la filosofía empresarial que considera al trabajador como un cliente interno de la organización, tiene como objetivo "vender" un determinado modelo de empresa a dicho empleado. A través de la venta de este modelo, se intenta incrementar su motivación, su participación y su orgullo de pertenencia, que se derivará en lo que se conoce como compromiso organizacional. Esto último a su vez es una de las formas en que las personas responden a su trabajo para alcanzar las metas de la organización.

Lechuga (1998) estima que cuando los colaboradores se encuentran en un ambiente de trabajo apropiado, tratarán de dar lo mejor de sí mismos, utilizando al máximo su potencial. Las mejores empresas se destacan por obtener resultados beneficiosos de su personal. 
Siguiendo en la misma línea, Arias (1991) manifiesta que un elemento complementario en el desarrollo de esta investigación es el de compromiso institucional, entendido como el deber moral adquirido hacia una persona o institución. Continuando con Arias, desde hace tiempo se ha establecido una distinción entre el contrato legal y el psicológico. Mientras una persona puede estar obligada formalmente a desempeñar una labor dentro de una organización, no necesariamente se liga afectivamente a ella. Puede trabajar con disgusto y sentir desprecio hacia la organización.

Trasladándonos a una institución del Estado y tomando en consideración la citas señaladas, el trabajo realizado respecto al marketing interno y el compromiso organizacional, a pesar de los avances realizados, todavía está lejos de alcanzar los objetivos propuestos. Los trabajadores no terminan de considerarse clientes internos en tanto perciben a la organización como separada de ellos, lo que se traduce en una falta de compromiso con ella.

En este sentido, y citando a Lagomarsino (2003), se requiere que los empleados desarrollen altos niveles de compromiso pues con ello se convierten en una fuente de innovación, asumen la iniciativa, tienen espíritu emprendedor, actúan proactivamente para mejorar la organización, asumen responsabilidades, transformándose de esa manera en una poderosa ventaja competitiva.

No obstante, si las personas son generadoras de los resultados organizacionales, entonces los empleados comprometidos constituyen una ventaja competitiva. Así, resulta trascendente para las organizaciones conocer cuáles son algunos de los factores sobre los cuales pueden influir para favorecer este compromiso.

Si bien se van operativizando algunos cambios como parte de un proceso de reconversión en cuanto a finalidades y responsabilidades, la correlación entre el marketing interno y el compromiso organizacional es limitada. Es por ello que el presente estudio busca entender el marketing interno y el compromiso que manifiestan los empleados de esta organización del Estado. De no superarse en su totalidad, lo más probable es que esta institución no logre cumplir con sus objetivos de manera exitosa.

Como consecuencia de lo expuesto, los problemas de la presente investigación se plantean de la siguiente manera:

¿Qué relación existe entre el marketing interno y el compromiso organizacional de los trabajadores de una empresa pública de Lima?

¿Qué relación existe entre las diversas dimensiones del marketing interno y las diversas dimensiones del compromiso organizacional de los trabajadores de una empresa pública de Lima?

¿Qué diferencias existen entre los trabajadores varones y mujeres de una empresa pública de Lima respecto del marketing interno? 
RELACIÓN ENTRE MARKETING INTERNO Y EL COMPROMISO ORGANIZACIONAL DE LOS TRABAJADORES DE UNA EMPRESA PÚBLICA DE LIMA

¿Qué diferencias existen entre los trabajadores varones y mujeres de una empresa pública de Lima respecto del compromiso organizacional?

¿Qué diferencias existen entre los trabajadores de una empresa pública de Lima, considerando su estado civil, respecto del marketing interno?

¿Qué diferencias existen entre los trabajadores de una empresa pública de Lima, considerando su estado civil, respecto del compromiso organizacional?

¿Qué diferencias existen entre los trabajadores de una empresa pública de Lima, considerando su rango de tiempo de servicios, respecto del marketing interno?

¿Qué diferencias existen entre los trabajadores de una empresa pública de Lima, considerando su rango de tiempo de servicios, respecto del compromiso organizacional?

¿Qué diferencias existen entre los trabajadores de una empresa pública de Lima, considerando su rango de edad, respecto del marketing interno?

¿Qué diferencias existen entre los trabajadores de una empresa pública de Lima, considerando su rango de edad, respecto del compromiso organizacional?

\section{Objetivos}

1. Determinar la relación que existe entre el marketing interno y el compromiso organizacional en los trabajadores de una empresa pública de Lima.

2. Establecer la relación que existe entre las diversas dimensiones del marketing interno y las diversas dimensiones del compromiso organizacional en los trabajadores de una empresa pública de Lima.

3. Determinar las diferencias que existen entre los trabajadores varones y mujeres de una empresa pública de Lima respecto del marketing interno.

4. Establecer las diferencias que existen entre los trabajadores varones y mujeres de una empresa pública de Lima respecto del compromiso organizacional.

5. Determinar las diferencias que existen entre los trabajadores de una empresa pública de Lima, considerando su estado civil, respecto del marketing interno.

6. Establecer las diferencias que existen entre los trabajadores de una empresa pública de Lima, considerando su estado civil, respecto del compromiso organizacional.

7. Determinar las diferencias que existen entre los trabajadores de una empresa pública de Lima, considerando su rango de tiempo de servicios, respecto del marketing interno. 
8. Establecer las diferencias que existen entre los trabajadores de una empresa pública de Lima, considerando su rango de tiempo de servicios, respecto del compromiso organizacional.

9. Determinar las diferencias que existen entre los trabajadores de una empresa pública de Lima, considerando su rango de edad, respecto del marketing interno.

10. Establecer las diferencias que existen entre los trabajadores de una empresa pública de Lima, considerando su rango de edad, respecto del compromiso organizacional.

\section{Hipótesis}

1. Existe una relación significativa entre el marketing interno y el compromiso organizacional en los trabajadores de una empresa pública de Lima.

2. Existen relaciones significativas entre las diversas dimensiones del marketing interno y las diversas dimensiones del compromiso organizacional en los trabajadores de una empresa pública de Lima.

3. Existen diferencias significativas entre los trabajadores varones y mujeres de una empresa pública de Lima respecto del marketing interno.

4. Existen diferencias significativas entre los trabajadores varones y mujeres de una empresa pública de Lima respecto del compromiso organizacional.

5. Existen diferencias significativas entre los trabajadores de una empresa pública de Lima, considerando su estado civil, respecto del marketing interno.

6. Existen diferencias significativas entre los trabajadores de una empresa pública de Lima, considerando su estado civil, respecto del compromiso organizacional.

7. Existen diferencias significativas entre los trabajadores de una empresa pública de Lima, considerando su rango de tiempo de servicios, respecto del marketing interno.

8. Existen diferencias significativas entre los trabajadores de una empresa pública de Lima, considerando su rango de tiempo de servicios, respecto del compromiso organizacional.

9. Existen diferencias significativas entre los de una empresa pública de Lima, considerando su rango de edad, respecto del marketing interno.

10. Existen diferencias significativas entre los trabajadores de una empresa pública de Lima, considerando su rango de edad, respecto del compromiso organizacional. 


\section{MÉTODO}

El método de investigación utilizado fue el método descriptivo; ya que además de describir las situaciones y eventos, mide diversos aspectos del fenómeno a investigar. El estudio descriptivo se desarrolla detallando las situaciones y eventos, es decir cómo se manifiesta el fenómeno a investigar, ya que este busca especificar las propiedades importantes del problema en cuestión. El método descriptivo mide independientemente los conceptos y también pueden ofrecer la posibilidad de predicciones aunque sean muy rudimentarias (Sánchez y Reyes, 2002).

\section{Diseño de investigación}

Para la selección del diseño de investigación se ha utilizado como base el libro de Hernández, Fernández y Baptista (2010) titulado "Metodología de la Investigación". Según estos autores, el diseño adecuado para esta investigación es de tipo descriptivo correlacional.

Su figura representativa es el siguiente:

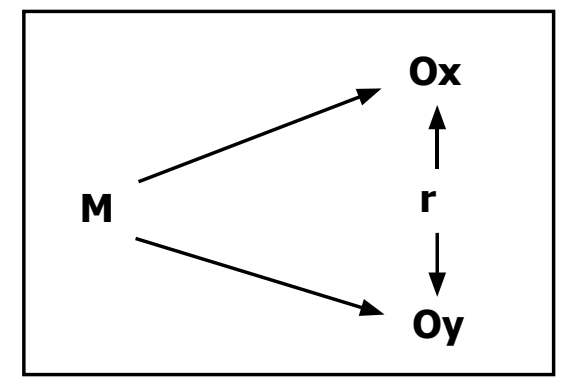

En la figura:

$\mathrm{M} \quad=$ Muestra de investigación

Ox, Oy $=$ Observaciones de las variables

$\mathrm{r} \quad=$ Relaciones entre variables

En nuestro caso, pretendemos relacionar el marketing interno y el compromiso organizacional en una muestra de trabajadores de una empresa pública de Lima.

\section{Muestra}

Para la determinación de una muestra representativa de los trabajadores de una empresa pública de Lima que fueron incorporados en el estudio, se ha calculado usando la fórmula para la determinación del tamaño de muestra para poblaciones finitas (Sierra Bravo, 1988), la que dio como resultado un total de 279 trabajadores distribuidos de la siguiente forma: 
Tabla 1. Composición general de la muestra

\begin{tabular}{ccc}
\hline Sexo & F & $\%$ \\
\hline Masculino & 172 & 61.6 \\
Femenino & 107 & 38.4 \\
\hline Edad & & \\
\hline $25-29$ & 25 & 9.0 \\
$30-34$ & 36 & 12.9 \\
$35-39$ & 53 & 19.0 \\
$40-44$ & 38 & 13.6 \\
$45-49$ & 54 & 19.4 \\
$50-54$ & 33 & 11.8 \\
$55-+$ & 40 & 14.3 \\
\hline
\end{tabular}

\section{Instrumentos}

Los instrumentos utilizados en el desarrollo de la presente investigación fueron el cuestionario de marketing interno, de María Bohnenberger, compuesto por cuatro subescalas: desarrollo, contratación y retención de los empleados, adecuación al trabajo y comunicación interna; y el inventario de compromiso organizacional, de Allen y Meyer, por tres subescalas: compromiso afectivo, compromiso normativo y compromiso continuo.

En ambos casos los resultados son calificados sobre la base de una escala Likert de cinco puntos, los cuales permitían expresar en términos de frecuencia la ocurrencia de los aspectos indicados y fluctúan desde muy en desacuerdo (1) para el menor valor, hasta muy de acuerdo (5) para la mayor valoración.

\section{Análisis de validez y confiabilidad de los instrumentos}

En la medida que se necesita garantizar la idoneidad de los instrumentos de evaluación, se procedió a realizar los análisis estadísticos respectivos que nos indiquen sus niveles de validez y confiabilidad. El análisis psicométrico del cuestionario de marketing interno indica que la prueba alcanza un alfa de Cronbach de 0.73 , lo que demuestra que el instrumento es confiable.

El análisis psicométrico del inventario de compromiso organizacional indica que la prueba alcanza un alfa de Cronbach de 0.94 , lo que demuestra que el instrumento es confiable.

Los análisis de la validez de los instrumentos, realizados a través del análisis factorial exploratorio, nos muestran que las pruebas presentan validez de constructo. 
RELACIÓN ENTRE MARKETING INTERNO Y EL COMPROMISO ORGANIZACIONAL DE LOS TRABAJADORES DE UNA EMPRESA PÚBLICA DE LIMA

\section{Resultados}

Tabla 2. Análisis de la bondad de ajuste de la curva normal de las variables estudiadas

\begin{tabular}{ccccc}
\hline VARIABLE & MEDIA & D.S. & K-S Z & SIG. \\
\hline Desarrollo & 15.41 & 2.23 & 1.77 & .004 \\
Contratación y retención de los empleados & 18.42 & 3.08 & 1.85 & .002 \\
Adecuación al trabajo & 14.18 & 2.73 & 1.37 & .045 \\
Comunicación interna & 32.97 & 4.85 & 1.22 & .100 \\
& & & & \\
Afectivo & 26.66 & 4.77 & 1.28 & .074 \\
\hline Normativo & 23.36 & 4.19 & 1.40 & .038 \\
Continuo & 30.63 & 5.59 & 1.36 & .049 \\
\hline
\end{tabular}

$\mathrm{n}=279$

Los resultados del análisis exploratorio de los datos (ver tabla 2), en lo que se refiere a la forma de distribución, efectuado a través del test de bondad de ajuste a la curva normal de Kolmogorov-Smirnov, indican que en todas las variables estudiadas se obtienen coeficientes K-S Z que son estadísticamente significativos, por lo que se puede concluir que no se presentan adecuadas aproximaciones a la curva normal y es factible utilizar, para el análisis de los datos, estadísticas no paramétricas.

Tabla 3. Análisis de correlación (Rho de Spearman) entre marketing interno y compromiso organizacional

\begin{tabular}{cc}
\hline Variables & Compromiso organizacional \\
Marketing interno & $0.79^{* * *}$ \\
\hline
\end{tabular}

$* \mathrm{p}<.05 * * \mathrm{p}<.01 * * * \mathrm{p}<.001$

$\mathrm{n}=279$

Los resultados presentados en la tabla 3 nos indican que existen correlaciones significativas entre los totales de las variables marketing interno y el compromiso organizacional $(\mathrm{r}=0.79)$.

Tabla 4. Análisis de correlación entre las dimensiones del marketing interno y las dimensiones compromiso organizacional

$\begin{array}{cccc}\text { Variables } & \text { Afectivo } & \text { Normativo } & \text { Continuo } \\ \text { Desarrollo } & 0.28^{* *} & 0.27^{* *} & 0.37 * * *\end{array}$




\begin{tabular}{cccc} 
Contratación y retención de los empleados & $0.39 * * *$ & $0.33 * *$ & $0.41 * * *$ \\
Adecuación al trabajo & $0.63^{* * *}$ & $0.53 * * * *$ & $0.52 * * *$ \\
Comunicación interna & $0.56 * * *$ & $0.50 * * *$ & $0.62 * * *$ \\
\hline
\end{tabular}

$* \mathrm{p}<.05 * * \mathrm{p}<.01 * * * \mathrm{p}<.001$

$\mathrm{N}=279$

Los resultados presentados en la tabla 4 nos indican que existen correlaciones significativas y positivas en todos los casos.

Tabla 5. Análisis comparativos de las áreas del marketing interno según el sexo en la muestra estudiada

\begin{tabular}{|c|c|c|c|c|c|c|}
\hline Variable & Sexo & $\mathrm{N}$ & $\begin{array}{c}\text { Rango } \\
\text { promedio }\end{array}$ & $\begin{array}{c}\text { U de Mann- } \\
\text { Whitney }\end{array}$ & Z & $\begin{array}{c}\text { Sig. } \\
\text { asintót. }\end{array}$ \\
\hline \multirow{3}{*}{ Desarrollo } & Masculino & 172 & 135.65 & \multirow{3}{*}{8453.00} & \multirow{3}{*}{-1.17} & \multirow{3}{*}{.243} \\
\hline & & & & & & \\
\hline & Femenino & 107 & 147.00 & & & \\
\hline \multirow{2}{*}{$\begin{array}{l}\text { Contratación y } \\
\text { retención }\end{array}$} & Masculino & 172 & 134.54 & \multirow{2}{*}{8262.50} & \multirow{2}{*}{-1.46} & \multirow{2}{*}{.145} \\
\hline & Femenino & 107 & 148.78 & & & \\
\hline \multirow{2}{*}{$\begin{array}{l}\text { Adecuación al } \\
\text { trabajo }\end{array}$} & Masculino & 172 & 118.39 & \multirow{2}{*}{5485.00} & \multirow{2}{*}{-5.72} & \multirow{2}{*}{$.000 * * *$} \\
\hline & Femenino & 107 & 174.74 & & & \\
\hline \multirow{2}{*}{$\begin{array}{c}\text { Comunicación } \\
\text { interna }\end{array}$} & Masculino & 172 & 120.40 & \multirow{2}{*}{5830.00} & \multirow{2}{*}{-5.17} & \multirow{2}{*}{$.000 * * *$} \\
\hline & Femenino & 107 & 171.51 & & & \\
\hline \multirow{3}{*}{ Total MI } & Masculino & 172 & 119.53 & \multirow{3}{*}{5681.00} & \multirow{3}{*}{-5.38} & \multirow{3}{*}{$.000 * * *$} \\
\hline & & & & & & \\
\hline & Femenino & 107 & 172.91 & & & \\
\hline
\end{tabular}

$* \mathrm{p}<.05 * * \mathrm{p}<.01 * * * \mathrm{p}<.001$

$\mathrm{N}=279$

El análisis comparativo del marketing interno por sexo (ver tabla 5) indica que existen diferencias estadísticas significativas en los siguientes casos:

- Adecuación al trabajo $(\mathrm{U}=5485.00 \mathrm{Z}=-5.72 \mathrm{p}<.001)$, notándose que la mayor calificación corresponde a las trabajadoras mujeres (Rango promedio $=$ 174.74) respecto de los varones (Rango promedio $=118.39$ ). 
RELACIÓN ENTRE MARKETING INTERNO Y EL COMPROMISO ORGANIZACIONAL DE LOS TRABAJADORES DE UNA EMPRESA PÚBLICA DE LIMA

- Comunicación interna $(\mathrm{U}=5830.00 \mathrm{Z}=-5.17 \mathrm{p}<.001)$, notándose que la mayor calificación corresponde a las trabajadoras mujeres (Rango promedio $=$ 171.51) respecto de los varones (Rango promedio $=120.40$ ).

- Total del marketing interno $(\mathrm{U}=5681.00 \mathrm{Z}=-5.38 \mathrm{p}<.001)$, notándose que la mayor calificación corresponde a las trabajadoras mujeres (Rango promedio $=$ 172.91) respecto de los varones (Rango promedio $=119.53$ ).

Tabla 6. Análisis comparativos de las áreas del compromiso organizacional según el sexo en la muestra estudiada

\begin{tabular}{|c|c|c|c|c|c|c|}
\hline Variable & Sexo & $\mathrm{N}$ & $\begin{array}{c}\text { Rango } \\
\text { promedio }\end{array}$ & $\begin{array}{l}\text { U de Mann- } \\
\text { Whitney }\end{array}$ & Z & Sig. asintót \\
\hline \multirow{2}{*}{ Afectivo } & Masculino & 172 & 119.56 & \multirow{2}{*}{5686.50} & \multirow{2}{*}{-5.39} & \multirow{2}{*}{$.000 * * *$} \\
\hline & Femenino & 107 & 172.86 & & & \\
\hline \multirow{2}{*}{ Normativo } & Masculino & 172 & 118.77 & \multirow{2}{*}{5550.50} & \multirow{2}{*}{-5.61} & \multirow{2}{*}{$.000 * * *$} \\
\hline & Femenino & 107 & 174.13 & & & \\
\hline \multirow[b]{2}{*}{ Continuo } & Masculino & 172 & 123.60 & \multirow[b]{2}{*}{6381.00} & \multirow[b]{2}{*}{-4.32} & \multirow[b]{2}{*}{$.000 * * *$} \\
\hline & Femenino & 107 & 166.36 & & & \\
\hline \multirow{2}{*}{ Total CO } & Masculino & 172 & 118.94 & \multirow{2}{*}{5579.50} & \multirow{2}{*}{-5.53} & \multirow{2}{*}{$.000 * * *$} \\
\hline & Femenino & 107 & 173.86 & & & \\
\hline
\end{tabular}

$* \mathrm{p}<.05 * * \mathrm{p}<.01 * * * \mathrm{p}<.001$

$\mathrm{N}=279$

El análisis comparativo del compromiso organizacional por sexo (ver tabla 6) indica que existen diferencias estadísticas significativas en los siguientes casos:

Compromiso afectivo $(\mathrm{U}=5686.50 \mathrm{Z}=-5.39 \mathrm{p}<.001)$, notándose que la mayor calificación corresponde a las trabajadoras mujeres $($ Rango promedio $=172.86$ ) respecto de los varones (Rango promedio $=119.56$ ).

Compromiso normativo $(\mathrm{U}=5550.50 \mathrm{Z}=-5.61 \mathrm{p}<.001)$, notándose que la mayor calificación corresponde a las trabajadoras mujeres (Rango promedio $=$ 174.13) respecto de los varones (Rango promedio $=118.77$ ).

Compromiso continuo $(\mathrm{U}=6381.00 \mathrm{Z}=-4.32 \mathrm{p}<.001)$, notándose que la mayor calificación corresponde a las trabajadoras mujeres (Rango promedio $=$ 166.36) respecto de los varones (Rango promedio $=123.60$ ). 
- Total compromiso organizacional $(\mathrm{U}=5579.50 \mathrm{Z}=-5.53 p<.001)$, notándose que la mayor calificación corresponde a las trabajadoras mujeres (Rango promedio $=173.86)$ respecto de los varones (Rango promedio $=118.94)$.

Tabla 7. Análisis de varianza por rangos de Kruskal - Wallis del marketing interno por estado civil

\begin{tabular}{c|c|c|c|c|c|c|c}
\hline \multicolumn{7}{c}{ Rango promedio } \\
Variables & $\begin{array}{c}\text { E. } \\
\text { civil }\end{array}$ & Soltero & Casado & Conv. & $\begin{array}{c}\text { Chi- } \\
\text { cuadrado }\end{array}$ & gl & $\begin{array}{c}\text { Sig. } \\
\text { asintót. }\end{array}$ \\
Desarrollo & $\mathrm{N}$ & 65 & 179 & 35 & & & $.000^{* * *}$ \\
Contratación y retención & 104.77 & 154.35 & 132.03 & 19.19 & & .626 \\
Adecuación al trabajo & 132.26 & 143.23 & 137.87 & 0.93 & & .182 \\
Comunicación interna & 132.29 & 141.85 & 144.83 & 0.82 & & .664 \\
Total marketing I & 127.00 & 148.51 & 120.64 & 5.71 & & .057 \\
\hline
\end{tabular}

$* \mathrm{p}<.05 * * \mathrm{p}<.01 * * * \mathrm{p}<.001$

$\mathrm{N}=279$

El análisis de varianza por rangos de Kruskall-Wallis del marketing interno por estado civil indica que existen diferencias estadísticas solo en el caso de desarrollo $($ Chi-Cuadrado $=19.19 \mathrm{gl}=2 \mathrm{p}<.001)$, notándose que los trabajadores casados (Rango promedio $=154.35)$ superan a los trabajadores solteros y convivientes.

Tabla 8. Análisis de varianza por rangos de Kruskal - Wallis del compromiso organizacional por estado civil

\begin{tabular}{c|c|c|c|c|c|c|c}
\hline & \multicolumn{9}{c}{ Rango promedio } \\
Variables & $\begin{array}{c}\text { E. } \\
\text { civil }\end{array}$ & Soltero & Casado & Conv. & $\begin{array}{c}\text { Chi- } \\
\text { cuadrado }\end{array}$ & gl & $\begin{array}{c}\text { Sig. } \\
\text { asintót. }\end{array}$ \\
C. afectivo & $\mathrm{N}$ & 65 & 179 & 35 & & & \\
C. normativo & 136.54 & 141.14 & 140.59 & .159 & & .924 \\
C. continuo & 139.55 & 142.82 & 126.39 & 1.234 & & .540 \\
Total CO & 131.09 & 143.49 & 138.71 & 1.144 & 2 & .564 \\
\hline
\end{tabular}

$* \mathrm{p}<.05 * * \mathrm{p}<.01 * * * \mathrm{p}<.001$

$\mathrm{N}=279$

El análisis de varianza por rangos de Kruskall-Wallis del compromiso organizacional por estado civil indica que no existen diferencias estadísticas significativas en caso alguno, por lo que los trabajadores, sin importar su estado civil, presentan las mismas características respecto a su compromiso organizacional. 
RELACIÓN ENTRE MARKETING INTERNO Y EL COMPROMISO ORGANIZACIONAL DE LOS TRABAJADORES DE UNA EMPRESA PÚBLICA DE LIMA

Tabla 9. Análisis de varianza por rangos de Kruskal - Wallis del marketing interno por tiempo de servicio

\begin{tabular}{c|c|c|c|c|c|c|c|c|c|c|c}
\hline & \multicolumn{7}{|c}{ Rango promedio } \\
Variables & Tiempo & $3-7$ & $8-12$ & $13-17$ & $18-22$ & $23-27$ & $28-32$ & $33-+$ & $\begin{array}{c}\text { Chi- } \\
\text { cuadrado }\end{array}$ & $\begin{array}{c}\text { Sig. } \\
\text { asintót. }\end{array}$ \\
$\mathrm{N}$ & 22 & 63 & 53 & 31 & 36 & 42 & 32 & & \\
Desarrollo & 160.45 & 142.26 & 154.66 & 123.21 & 127.68 & 160.08 & 100.97 & 16.15 & & $.013^{* *}$ \\
Contratación y retención & 157.50 & 128.88 & 155.40 & 115.24 & 122.25 & 153.62 & 150.44 & 10.90 & & .091 \\
Adecuación al trabajo & 167.23 & 130.13 & 144.21 & 132.65 & 150.00 & 111.58 & 166.92 & 13.39 & 6 & $.037^{*}$ \\
Comunicación interna & 153.73 & 108.62 & 142.65 & 128.65 & 174.00 & 141.01 & 159.38 & 19.24 & & $.004^{* *}$ \\
Total marketing I & 157.82 & 122.77 & 151.88 & 119.44 & 161.32 & 132.52 & 147.75 & 10.31 & & .112 \\
\hline
\end{tabular}

$* \mathrm{p}<.05 * * \mathrm{p}<.01 * * * \mathrm{p}<.001$

$\mathrm{N}=279$

El análisis de varianza por rangos de Kruskall-Wallis del marketing interno por tiempo de servicios indica que existen diferencias estadísticas significativas en los siguientes casos:

Desarrollo (Chi-Cuadrado $=16.15 \mathrm{gl}=6 \mathrm{p}<.01)$, notándose que los trabajadores que se encuentran entre los 3 y 7 años de tiempo de servicios (Rango promedio $=$ 160.45) y los 28 y 32 años de tiempo de servicios (Rango promedio $=160.08$ ) superan a los trabajadores de los otros rangos de tiempo de servicios.

Adecuación al trabajo (Chi-Cuadrado $=13.39 \mathrm{gl}=6 \mathrm{p}<.05$ ), notándose que los trabajadores que se encuentran entre los 3 y 7 años de tiempo de servicios (Rango promedio $=167.23$ ) y los 33 a más años de tiempo de servicios (Rango promedio $=166.92$ ) superan a los trabajadores de los otros rangos de tiempo de servicios.

Comunicación interna (Chi-Cuadrado $=19.24 \mathrm{gl}=6 \mathrm{p}<.01$ ), notándose que los trabajadores que se encuentran entre los 23 y 27 años de tiempo de servicios ( Rango promedio $=174.00$ ) y los 33 a más años de tiempo de servicios (Rango Promedio $=159.38$ ) superan a los trabajadores de los otros rangos de tiempo de servicios.

Tabla $\mathbf{N}^{0}$ 10. Análisis de varianza por rangos de Kruskal - Wallis del compromiso organizacional por tiempo de servicios

\begin{tabular}{ccccccccccc}
\hline \multicolumn{8}{c}{ Rango promedio } \\
Variables & Tiempo & $3-7$ & $8-12$ & $13-17$ & $18-22$ & $23-27$ & $28-32$ & $33-+c_{\text {Chi- }}^{\text {cuadrado gl }} \begin{array}{c}\text { Sig. } \\
\text { asintót. }\end{array}$ \\
& $\mathrm{N}$ & 22 & 63 & 53 & 31 & 36 & 42 & 32
\end{tabular}




\begin{tabular}{cccccccccc} 
C. afectivo & 178.91 & 141.13 & 129.42 & 139.16 & 166.17 & 98.77 & 154.03 & 21.94 & $.001 * *$ \\
C. normativo & 157.23 & 163.55 & 142.67 & 101.19 & 154.69 & 114.94 & 131.33 & 19.47 & $.003 * *$ \\
C. continuo & 169.27 & 144.80 & 144.09 & 125.45 & 156.24 & 109.31 & 139.75 & 11.89 & 6 \\
Total CO & 171.18 & 147.69 & 139.11 & 122.05 & 163.60 & 104.23 & 142.69 & 16.81 & .065 \\
\hline
\end{tabular}

$* \mathrm{p}<.05 * * \mathrm{p}<.01 * * * \mathrm{p}<.001$

$\mathrm{N}=279$

El análisis de varianza por rangos de Kruskall-Wallis del marketing interno por tiempo de servicios indica que existen diferencias estadísticas significativas en los siguientes casos:

Compromiso afectivo (Chi-Cuadrado $=21.94 \mathrm{gl}=6 \mathrm{p}<.01$ ), notándose que los trabajadores que se encuentran entre los 3 y 7 años de tiempo de servicios (Rango promedio $=178.91$ ) y los 23 y 27 años de tiempo de servicios (Rango promedio $=166.17$ ) superan a los trabajadores de los otros rangos de tiempo de servicios.

Compromiso normativo (Chi-Cuadrado $=19.47 \mathrm{gl}=6 \mathrm{p}<.01$ ), notándose que los trabajadores que se encuentran entre los 8 y 12 años de tiempo de servicios (Rango promedio $=163.55)$ y los 3 y 7 años de tiempo de servicios (Rango promedio $=157.23$ ) superan a los trabajadores de los otros rangos de tiempo de servicios.

Total del compromiso organizacional (Chi-Cuadrado $=16.81 \mathrm{gl}=6 \mathrm{p}<.01$ ), notándose que los trabajadores que se encuentran entre los 3 y 7 años de tiempo de servicios (Rango promedio $=171.18$ ) y los 23 y 27 años de tiempo de servicios (Rango promedio $=163.60$ ) superan a los trabajadores de los otros rangos de tiempo de servicios.

Tabla 11. Análisis de varianza por rangos de Kruskal - Wallis del marketing interno por rango de edad

\begin{tabular}{|c|c|c|c|c|c|c|c|c|c|c|c|}
\hline \multirow[b]{2}{*}{ Variables } & \multicolumn{8}{|c|}{ Rango promedio } & \multirow{2}{*}{$\begin{array}{l}\text { Chi- } \\
\text { cuadrado }\end{array}$} & \multirow[b]{2}{*}{$\mathrm{gl}$} & \multirow{2}{*}{$\begin{array}{l}\text { Sig. } \\
\text { asintót. }\end{array}$} \\
\hline & $\begin{array}{c}\text { Edad } \\
\mathrm{N}\end{array}$ & $\begin{array}{c}25-29 \\
25\end{array}$ & $\begin{array}{c}30-34 \\
36\end{array}$ & $\begin{array}{c}35-39 \\
53\end{array}$ & $\begin{array}{c}40-44 \\
38\end{array}$ & $\begin{array}{c}45-49 \\
54\end{array}$ & $\begin{array}{c}50-54 \\
33\end{array}$ & $\begin{array}{c}55-+ \\
40\end{array}$ & & & \\
\hline \multicolumn{2}{|c|}{ Desarrollo } & 133.22 & 146.43 & 128.49 & 137.36 & 159.18 & 123.52 & 143.93 & 6.30 & & .390 \\
\hline \multicolumn{2}{|c|}{$\begin{array}{l}\text { Contratación y } \\
\text { retención }\end{array}$} & 154.16 & 150.24 & 129.66 & 139.62 & 115.27 & 138.94 & 170.26 & 13.35 & & $.038^{*}$ \\
\hline \multicolumn{2}{|c|}{$\begin{array}{c}\text { Adecuación al } \\
\text { trabajo }\end{array}$} & 172.58 & 130.11 & 140.68 & 157.34 & 106.20 & 131.89 & 163.48 & 19.88 & 6 & $.003 * *$ \\
\hline \multicolumn{2}{|c|}{$\begin{array}{c}\text { Comunicación } \\
\text { interna }\end{array}$} & 136.42 & 147.47 & 114.78 & 157.05 & 161.00 & 110.03 & 149.10 & 16.08 & & $.013 * *$ \\
\hline \multicolumn{2}{|c|}{ Total marketing I } & 150.22 & 146.11 & 131.58 & 141.57 & 137.43 & 123.24 & 155.08 & 4.08 & & .665 \\
\hline \multicolumn{12}{|c|}{$* \mathrm{p}<.05 * * \mathrm{p}<.01 * * * \mathrm{p}<.001$} \\
\hline \multicolumn{11}{|c|}{ ReVISTA de InVEstigación en Psicología - Vol. 17, N. ${ }^{0} 1$} & 221 \\
\hline
\end{tabular}


El análisis de varianza por rangos de Kruskall-Wallis del marketing interno por rango de edad indica que existen diferencias estadísticas significativas en los siguientes casos:

Contratación y retención de los empleados (Chi-Cuadrado $=13.55 \mathrm{gl}=6 \mathrm{p}<$ .05), notándose que los trabajadores que se encuentran entre los 55 y más años de edad (Rango promedio $=170.26)$ y los 25 y 29 años de edad (Rango promedio $=154.16$ ) superan a los trabajadores de los otros rangos de edad.

Adecuación al trabajo (Chi-Cuadrado $=19.88 \mathrm{gl}=6 \mathrm{p}<.01)$, notándose que los trabajadores que se encuentran entre los 25 y 29 años de edad (Rango promedio $=172.58)$ y los 55 y más años de edad (Rango promedio $=163.48)$ superan a los trabajadores de los otros rangos de edad.

Comunicación interna (Chi-Cuadrado $=16.08 \mathrm{gl}=6 \mathrm{p}<.01$ ), notándose que los trabajadores que se encuentran entre los 45 y 49 años de edad (Rango promedio $=161.00)$ y los 40 y 44 años de edad (Rango promedio $=157.05)$ superan a los trabajadores de los otros rangos de edad.

Tabla 12. Análisis de varianza por rangos de Kruskal - Wallis del compromiso organizacional por rango de edad

\begin{tabular}{cccccccccccc}
\hline & \multicolumn{8}{c}{ Rango promedio } & & & Chi- \\
Variables & Edad & $25-29$ & $30-34$ & $35-39$ & $40-44$ & $45-49$ & $50-54$ & $55-+$ & $\begin{array}{c}\text { Sig. } \\
\text { cuadrado } \\
\text { asintót. }\end{array}$ & \\
& $\mathrm{N}$ & 25 & 36 & 53 & 38 & 54 & 33 & 40 & & \\
C. afectivo & 145.88 & 164.49 & 127.71 & 151.57 & 113.47 & 148.17 & 148.66 & 12.19 & .058 \\
C. normativo & 158.06 & 156.71 & 156.37 & 125.47 & 123.10 & 124.02 & 141.79 & 10.02 & .124 \\
C. continuo & 141.54 & 151.31 & 130.39 & 153.34 & 139.55 & 141.56 & 128.25 & 3.39 & .758 \\
Total CO & 150.64 & 159.93 & 133.84 & 142.51 & 126.50 & 140.00 & 139.41 & 4.50 & .609 \\
\hline
\end{tabular}

$* \mathrm{p}<.05 * * \mathrm{p}<.01 * * * \mathrm{p}<.001$

$\mathrm{N}=279$

El análisis de varianza por rangos de Kruskall-Wallis del marketing interno por rango de edad indica que no existen diferencias estadísticas significativas en caso alguno.

\section{DISCUSIÓN}

Los análisis psicométricos a los que fue sometido el inventario de marketing interno revelan que todos los ítems forman parte de dicho inventario y corresponden a cada una de las cuatro escalas asignadas previamente. El instrumento es confiable, en la medida que sus escalas obtuvieron coeficientes alfa de Cronbach con valores que oscilan entre 0.69 y 0.77 , obteniendo además un alfa de Cronbach total para toda la prueba de 0.73 . 
En cuanto a la validez, el resultado del análisis factorial exploratorio indica que la prueba está conformada por un solo factor que explica el $76.75 \%$ de la varianza total. Alcanza además un valor de 0.75 en la medida de adecuación del muestreo de Kaiser-Meyer-Olkin, el mismo que puede considerarse como adecuado. Estos resultados nos indican que la prueba tiene validez de constructo.

En lo que se refiere a los análisis realizados para demostrar la confiabilidad del inventario de compromiso organizacional, revelan que el instrumento es confiable en la medida que el coeficiente alfa de Cronbach de toda la prueba llega a 0.94, lo que indica que toda la prueba es confiable.

El análisis de la validez de constructo, que se realizó a través del análisis factorial exploratorio aplicando el método de los componentes principales, permite apreciar que la prueba de compromiso organizacional presenta una medida de adecuación del muestreo de Kaiser-Meyer-Olkin que asciende a 0.77; el cual puede clasificarse como adecuado. Además presenta un test de esfericidad de Bartlett cuyo valor denota que la matriz de correlaciones entre áreas es significativa. La varianza explicada alcanza un nivel de $90.30 \%$. Estos hallazgos indican que la prueba tiene validez de constructo.

En lo que respecta a la hipótesis general de investigación -“Existe una relación significativa entre el marketing interno y el compromiso organizacional en los trabajadores de una empresa pública de Lima"-, los resultados obtenidos indican que existen correlaciones entre las variables en estudio, por lo que podemos afirmar que la citada hipótesis ha sido respaldada. Esto muestra que estos resultados están en la misma línea que los encontrados por los diversos autores que se expusieron en los antecedentes y además confirmaría las propuestas teóricas de Mc Carthy, la Asociación Americana de Marketing (AMA) y Mayer y Allen que hemos expuesto en nuestro marco teórico, quienes resaltan la trascendencia de las variables estudiadas.

Se debe entender que para desplegar un marketing interno efectivo es esencial conformar un plan de marketing dirigido a los empleados de la organización, al cual se le debe dar tanta atención como al plan dirigido al cliente externo, ya que si no logramos que el personal haga suyos los intereses de la entidad, nuestra labor externa no será lo suficientemente efectiva y no obtendremos los resultados deseados.

Uno de los mejores resultados de la aplicación del marketing interno es cuando los trabajadores se sienten "dueños" del negocio y son recompensados correctamente por la lealtad que tienen ante su empresa. Esto porque se sienten motivados y son escuchados cuando se les brinda canales de comunicación fluida. En la medida que se brinde mayor importancia a los clientes internos, estos sentirán mayor obligación para dar importancia a los clientes externos y, por ende, estarán dispuestos a sobrepasar sus expectativas.

Las organizaciones económicas necesitan investigar y conocer a fondo lo que sucede dentro de su ámbito interno para después actuar en consecuencia con la 
información obtenida y trazar la estrategia de marketing interno más adecuada. Muchas de estas entidades no han logrado aún concientizar la importancia del marketing interno, colocando sus recursos humanos al mismo nivel de los recursos monetarios o físicos.

Las empresas ya no están solamente en competencia por conseguir y retener clientes externos, sino también por clientes internos. Son los empleados comprometidos -aquellos que logran identificar sus competencias, medir sus resultados y que buscan satisfacer siempre las necesidades del cliente externo- quienes se convierten en parte fundamental de la cadena de valor, que llega hasta conseguir la fidelidad de los usuarios o consumidores.

El compromiso organizacional es otro de los mecanismos que tienen las instituciones para analizar la lealtad y vinculación de los empleados con su organización. En este aspecto podemos observar que los niveles de compromiso que manifiestan los trabajadores no son lo importantes que se desearían, por lo que debemos insistir en proporcionarles mejores condiciones de trabajo. De esta manera podremos incrementar estos niveles de compromiso, necesarios para el buen funcionamiento de una institución tan importante como la que hemos analizado.

\section{CONCLUSIONES}

1. Los análisis de las correlaciones entre el marketing interno y el compromiso organizacional, presentados en la tabla 10 , indican que existen correlaciones significativas entre los totales de estas variables.

2. Los análisis de las correlaciones entre las diversas dimensiones del marketing interno y las diversas dimensiones del compromiso organizacional, presentados en la tabla 11, indican que existen correlaciones significativas y positivas.

3. El análisis comparativo del marketing interno por sexo (tabla 12) indica que existen diferencias estadísticas significativas en los casos de adecuación al trabajo, comunicación interna y en el total del marketing interno, apreciándose que las trabajadoras mujeres alcanzan mayores puntajes que los varones.

4. El análisis comparativo del compromiso organizacional por sexo (tabla 13) indica que existen diferencias estadísticas significativas en los casos de compromiso afectivo, compromiso normativo, compromiso continuo y en el total del compromiso organizacional, apreciándose que las trabajadoras mujeres alcanzan mayores puntajes que los varones.

5. El análisis comparativo del marketing interno por estado civil (tabla 14) indica que existen diferencias estadísticas significativas solo en el caso de desarrollo, apreciándose que los trabajadores casados superan a los trabajadores solteros y convivientes. 
6. El análisis comparativo del compromiso organizacional por estado civil (tabla 15), indica que no existen diferencias estadísticas significativas en caso alguno.

7. El análisis comparativo del marketing interno por tiempo de servicios (tabla 16) indica que existen diferencias estadísticas significativas en los casos de desarrollo, adecuación al trabajo y comunicación interna, apreciándose que los trabajadores de 3 y 7 y de 23 y 27 años de tiempo de servicios superan a los trabajadores de otros rangos de tiempo de servicios.

8. El análisis comparativo del compromiso organizacional por tiempo de servicios (tabla 17) indica que existen diferencias estadísticas significativas en los casos de compromiso afectivo, compromiso normativo y en el total del compromiso organizacional, apreciándose que los trabajadores de 3 y 7 años de tiempo de servicios superan a los trabajadores de otros rangos de tiempo de servicios.

9. El análisis comparativo del marketing interno por rango de edad (tabla 18) indica que existen diferencias estadísticas significativas en los casos de contratación y retención de los empleados, adecuación al trabajo y comunicación interna, apreciándose que los trabajadores de 45 y 49 y de 25 y 29 años de tiempo de servicios superan a los trabajadores de otros rangos de edad.

10. El análisis comparativo del compromiso organizacional por rango de edad (tabla 19) indica que no existen diferencias estadísticas significativas en caso alguno.

\section{REFERENCIAS BIBLIOGRÁFICAS}

American Marketing Association, AMA (1985). AMA approves new definition. Marketing News, 19 March. Citado por Cadavid, H. (2004). Marketing de emociones. La forma para lograr fidelidad de los clientes. Semestre Económico, vol. 7, núm. 13, enerojunio, 2004, pp. 203-211. Redalyc.

Arias, F. (1991). Administración de recursos humanos. México: Trillas.

Arias, F. (2001). El compromiso personal hacia la organización y la intención de permanencia: algunos factores para su incremento. Revista Contaduría y Administración, 200, 5-12.

Arciniega, L. M. (2002). Compromiso Organizacional en México: ¿Cómo hacer que la gente se ponga la camiseta? Dirección Estratégica, Revista de Negocios del ITAM, 2(1), 21-23.

Bohnenberger, M. C. (2005). Marketing interno: la actuación conjunta entre recursos humanos y marketing en busca del compromiso organizacional. Tesis doctoral. Universidad de las Islas Baleares.

Coca, C. y Milton, A. (2008), El concepto de Marketing: pasado y presente. Revista de Ciencias Sociales, Vol. 14 (2), pp. 391-412 
RELACIÓN ENTRE MARKETING INTERNO Y EL COMPROMISO ORGANIZACIONAL DE LOS TRABAJADORES DE UNA EMPRESA PÚBLICA DE LIMA

Horovitz, J. y Jurgens, M. (1993). La satisfacción total del cliente. Madrid: McGraw Hill.

Hernández y otros (2010) Metodología de la Investigación. Ed. Mc Graw Hill. México.

Kotler, P. y Levy, S.J. (1969). Broadening the Concept of Marketing. Journal of Marketing. Vol. 33, N ${ }^{0}$ 1, January. Pp. 10-15. Citado por Coca, C. y Milton, A. (2008), El concepto de Marketing: pasado y presente. Revista de Ciencias Sociales, Vol. 14 (2), pp. 391-412.

Kotler, P. (1992). Dirección de Marketing. Editorial Prentice Hall, España.

Kotler, P. y Keller, K. (2006). Dirección de Marketing. México: Ed. Pearson

Lechuga Santillán E. (1998), Estrategias para la optimización de los Recursos Humanos. Grupo Editorial ISEF, México.

Lagomarsino, Rául (2003). Compromiso organizacional. Revista de Antiguos Alumnos, Año VI, No. 2, No. pp. 79-83

Meyer, J.P., Allen, N.J. y Smith, C.A. (1993). Commitment to organizations and occupations: Extension and tests of a three-component conceptualization. Journal of Applied Psychology, 78, 538-551.

Robbins, S. P. (2004). Comportamiento Organizacional. (México: Prentice Hall.

Stanton, W. (2007). Fundamentos de Marketing. Madrid. Editorial Mc Graw Hill Interamericana.

Sánchez y Reyes, (2002). Metodología y diseños en la Investigación Científica. Lima. Universidad Ricardo Palma.

Saracco, C. (2004), La función estratégica del marketing. Revista Harvard Deusto. Marketing y Ventas, Vol. 65 (11-12), pp. 32-35.

Varona Madrid, Federico (1993). Conceptualización y supervisión de la comunicación y el compromiso organizacional, en Diálogos de la comunicación, No. 35, pp. 68-77 